\title{
Computational studies of the effects of myocardial blood flow reductions on cardiac metabolism Jennifer E Salem ${ }^{1,3}$, William C Stanley² and Marco E Cabrera*1,2,3
}

\author{
Address: ${ }^{1}$ Department of Biomedical Engineering, Case Western Reserve University, Cleveland, OH, USA, ${ }^{2}$ Department of Physiology and \\ Biophysics, Case Western Reserve University, Cleveland, OH, USA and ${ }^{3}$ Department of Pediatrics, Rainbow Babies and Children's Hospital, \\ Cleveland, OH, USA \\ Email: Jennifer E Salem - jec9@po.cwru.edu; William C Stanley - wcs4@po.cwru.edu; Marco E Cabrera* - mec6@po.cwru.edu \\ * Corresponding author
}

Published: 02 June 2004

BioMedical Engineering OnLine 2004, 3:15
Received: 02 March 2004

Accepted: 02 June 2004

This article is available from: http://www.biomedical-engineering-online.com/content/3/1/15

(C) 2004 Salem et al; licensee BioMed Central Ltd. This is an Open Access article: verbatim copying and redistribution of this article are permitted in all media for any purpose, provided this notice is preserved along with the article's original URL.

\begin{abstract}
Background: A computational model of myocardial energy metabolism was used to assess the metabolic responses to normal and reduced myocardial blood flow. The goal was to examine to what extent glycolysis and lactate formation are controlled by the supply of glycolytic substrate and/or the cellular redox (NADH/NAD $)$ and phosphorylation (ATP/ADP) states.

Methods: Flow was reduced over a wide range and for a sufficient duration in order to investigate the sequence of events that occur during the transition to a new metabolic steady state.

Results: Simulation results indicated multiple time-dependent controls over both glycolysis and lactate formation.

Conclusions: Changes in phosphorylation state and glucose uptake only significantly affect the initial phase of the glycolytic response to ischemia, while glycogen breakdown exerts control over glycolysis during the entire duration of ischemia. Similarly, changes in the redox state affect the rates of lactate formation and release primarily during the initial transient phase of the response to the reductions in blood flow, while the rate of glycolysis controls the rate of lactate formation throughout the entire period of adaptation.
\end{abstract}

\section{Background}

The primary effect of reduced myocardial blood flow is a decrease in the rate of aerobic ATP resynthesis, an increase in the cellular redox state (NADH/NAD+), acceleration of glycogenolysis and glycolysis, accumulation of lactate, and a switch from net lactate uptake to net lactate release by the myocardium. Studies in pigs and dogs have shown that the extent of the metabolic derangement (e.g., increased glycolytic rate and lactate production) during reduced myocardial blood flow is dependent upon the severity and duration of flow reduction [1-6]. Glucose uptake increases during moderate ischemia, but decreases in severely ischemic myocardium due to limited glucose delivery $[7,8]$, causing a greater reliance on glycogen for glycolytic substrate. The classic switch to net lactate release with the onset of reduced myocardial blood flow (30$50 \%$ reduction) gradually reverses back to no net lactate exchange after 40 to 120 minutes [9]. The control mechanisms responsible for the gradual reduction in lactate release are unclear.

At a given demand, $\mathrm{O}_{2}$ delivery to the myocardium determines the rate of oxidative phosphorylation, which sets the cellular NADH/NAD ${ }^{+}$and ATP/ADP ratios, both of which control the rate of glycolysis and lactate formation [10]. Ischemia results in impaired oxidative 
phosphorylation and a decrease in the ATP/ADP and phosphocreatine/creatine $(\mathrm{PCr} / \mathrm{Cr}$ ) ratios, which accelerate ATP formation by glycolysis. The rate of glycolysis is also dependent upon substrate supply (i.e., myocardial glycogen concentration and the uptake of exogenous glucose) [11]. It is unclear to what extent the rates of glycolysis and lactate formation, in the transition from normal to ischemic conditions, are controlled by the supply of glycolytic substrate or the cellular redox and phosphorylation states (NADH/NAD ${ }^{+}$and ATP/ADP). Dynamic information characterizing some of the mechanisms that control the rate of glycolysis could be obtained from examination of transient metabolic responses to sudden reductions in myocardial blood flow of various magnitudes and durations. However, relevant in vivo studies examining the inter-regulation of oxidative and glycolytic metabolism during the normal-to-reduced flow transition have not been performed over a wide range of myocardial blood flows during prolonged periods of ischemia. Moreover, it is extremely difficult to continuously measure key metabolic processes in vivo with current experimental techniques, especially during the first few minutes of this transition. In particular, tissue biopsies are taken at least 10 minutes after the onset of ischemia, which is already past the period when dynamics changes occur. The characteristics of metabolic control mechanisms, typically elucidated during the transients, are then unattainable. An alternative approach to animal or human experimentation is to use computational models to predict the metabolic responses and to analyze covariations in the dynamics of key concentrations and flux rates and their interdependence at various degrees of ischemia [12-16].

We recently developed a computational model of cardiac metabolism for large mammals [17]. The model was validated under steady-state conditions with normal and reduced myocardial flow (60\% reduction). Specifically, the model predicted changes in (1) lactate, glycogen, acetyl-CoA, CoA, ATP, and PCr concentrations, (2) glucose, fatty acid, and lactate uptakes, and (3) contribution of fatty acids and carbohydrates to acetyl-CoA formation and was validated with published experimental data preand post-induction of moderate ischemia. Unfortunately, to date, it has not been possible to validate the transient changes in fluxes and concentrations due to lack of appropriate in vivo experimental data. The main goal of the present investigation was to determine to what extent glycolysis and lactate formation, in the transition from normal to ischemic conditions, are regulated by the fluxes supplying glycolytic substrate or the cellular redox and phosphorylation states. To accomplish this, we extended our previous model and simulated the metabolic responses to step decreases in blood flow in order to examine the transition to a new metabolic steady state. Specifically, computer simulations of the metabolic responses to myocardial blood flow reductions of varying degrees and durations were performed to investigate the dynamics of key metabolite concentrations and fluxes from the onset of ischemia out to 6 hours.

\section{Methods}

To quantitatively investigate the effects of magnitude and duration of a reduction in myocardial blood flow on cardiac metabolism, we made some modifications to our previous computational model [17] and simulated the metabolic responses to a wide range of blood flow reductions (from mild to very low-flow ischemia). Presented in this work are the metabolic responses to various graded reductions in myocardial blood flow (MBF), focusing on the substrate concentrations and fluxes related to pyruvate metabolism (Fig. 1). The model consists of a set of interdependent dynamic mass balance equations, one for each species included. The general form of the mass balance (equation 1), shown for species $j$, is described by:

$\frac{d C_{j}}{d t}=P_{j}^{*}-U_{j}^{*}+\operatorname{MBF}\left(C_{a, j}-\sigma_{j} C_{j}\right)$

where all terms are normalized per amount of tissue under the assumption that the density of both tissue and blood are $1 \mathrm{~g} \mathrm{ml}^{-1}$. Here $P_{j}^{*}$ and $U_{j}{ }^{*}$ are the specific rates of production and utilization of species $j$. The last term on the right side represents the tissue-blood exchange where $\mathrm{MBF}$ is the specific blood flow to the heart, $\sigma_{j}$ is the bloodtissue partition coefficient, and $C_{a, j}$ is the arterial blood concentration. In this single organ model, the arterial concentrations are assumed to remain constant since the relative contribution of the cardiac venous effluent to the pool of the metabolites present in the blood is very small, even during ischemia, as evidenced by in vivo experimental preparations $[18,19]$. The production and utilization rates of each species depend on all of the reactions included in the model that result in the formation or consumption of the species, respectively, and are determined from reaction fluxes with appropriate stoichiometric coefficients $\left(\beta_{k j}\right)$ where $\mathrm{p}$ refers to the reaction process (equation 2).

$$
P_{j}-U_{j}=\sum_{k=1}^{n} \beta_{k j} \phi_{k j}^{p}-\sum_{k=1}^{m} \beta_{j k} \phi_{j k}^{p}
$$

In the current model, the effective-volume coefficient is eliminated by normalizing concentrations and flux rates. In addition, the flux rates $\left(\Phi_{k j}\right)$ are expressed as MichaelisMenten functions of the reactant concentration, $C_{k^{\prime}}$ instead of following first-order kinetics as presented previously [17]:

$\phi_{k j}=V_{k j} \frac{C_{k}}{K_{m}+C_{k}}$ 


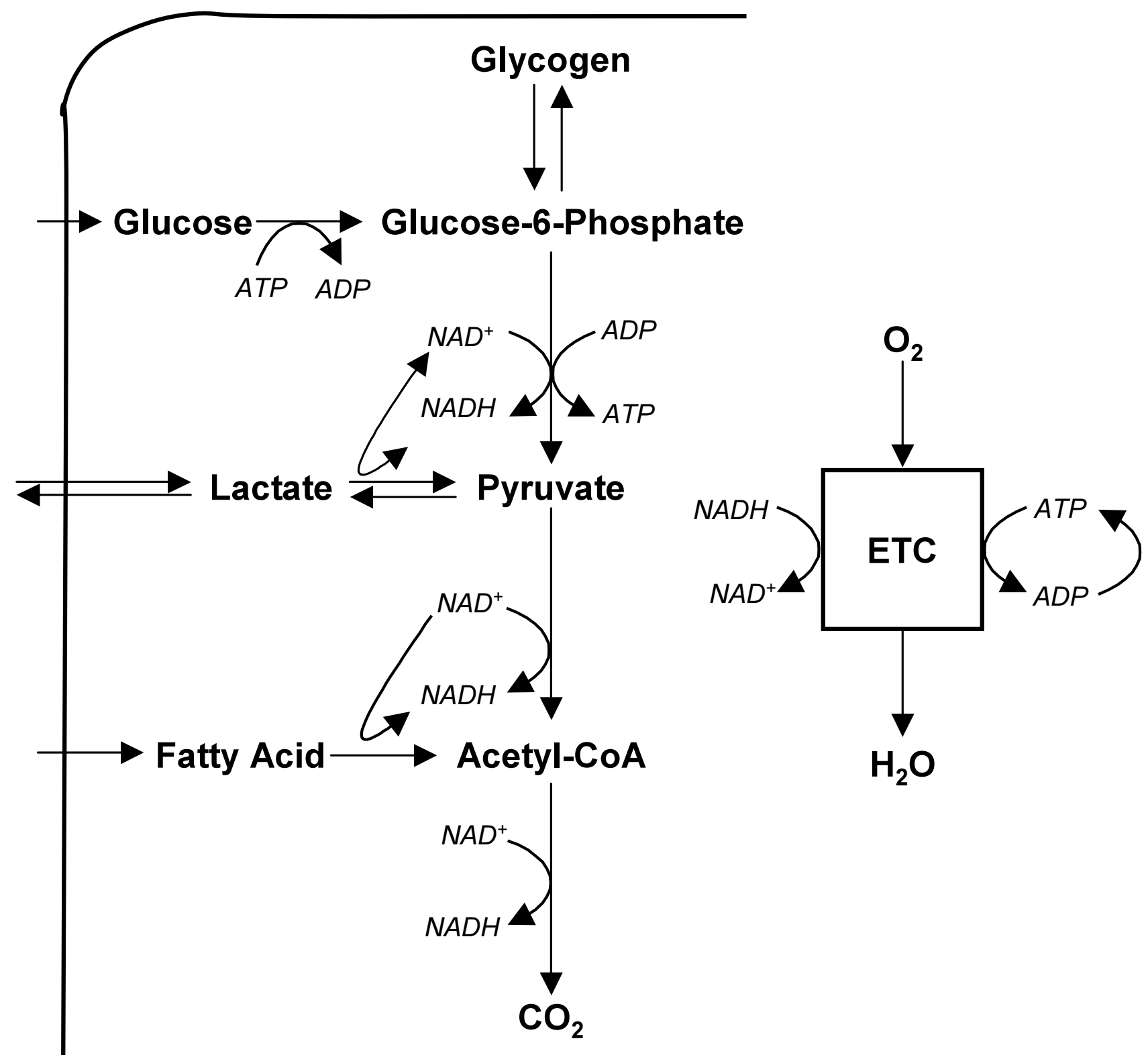

Figure I

Schematic of metabolic pathways.

In the flux expressions, $V_{k j}$ replaces the rate coefficient, $\lambda_{j k}$ of the previous version of the model, and represents the control exerted by the regulatory metabolites, which include $\mathrm{Cr} / \mathrm{PCr}, \mathrm{NADH} / \mathrm{NAD}^{+}, \mathrm{ADP} / \mathrm{ATP}$, and acetyl-CoA/ free-CoA. The specific $V_{k j}$ representations are presented in Table 1 , where $\mathrm{CS}=\mathrm{CR} / \mathrm{PCr}, \mathrm{RS}=\mathrm{NADH} / \mathrm{NAD}^{+}, \mathrm{PS}=$ $\mathrm{ADP} / \mathrm{ATP}$, and $\mathrm{AF}=$ acetyl-CoA/free-CoA. The flux rates, $\Phi_{k j}$ are expressed in a Michaelis-Menten form so that the reaction rates can respond rapidly to small changes in sub- strate concentrations or regulatory metabolites [20] thus allowing for feedback in the form of mass action (Table 1). With an increase in the product concentration the flux involving the formation of that product decreases. Similarly, with an increase in substrate concentration the flux increases in the direction of product formation.

In addition, pyruvate exchange between the blood and myocardium was included in the current model, although 
Table I: Rate Coefficient Expressions

$$
\begin{aligned}
& V_{G L \rightarrow G P}=V_{\max , G L \rightarrow G P} \times\left(\frac{C S}{C S_{0}+C S}\right) \\
& V_{F A \rightarrow T G}=V_{\max , F A \rightarrow T G} \times\left(\frac{P S^{-1}}{P S_{0}^{-1}+P S^{-1}}\right) \\
& V_{F A \rightarrow A C}=V_{\max , F A \rightarrow A C} \times\left[\varepsilon\left(\frac{A F^{-1}}{A F_{0}^{-1}+A F^{-1}}\right)+(1-\varepsilon)\left(\frac{R S^{-1}}{R S_{0}^{-1}+R S^{-1}}\right)\right] ; \quad \varepsilon=0.6 \\
& V_{G P \rightarrow P Y}=V_{\max , G P \rightarrow P Y} \times\left(\frac{P S}{P S_{0}+P S}\right) \\
& V_{G P \rightarrow G Y}=V_{\max , G P \rightarrow G Y} \times\left[\varepsilon\left(\frac{P S^{-1}}{P S_{0}^{-1}+P S^{-1}}\right)+(1-\varepsilon)\left(\frac{C S^{-1}}{C S_{0}^{-1}+C S^{-1}}\right)\right] ; \varepsilon=0.75 \\
& V_{G Y \rightarrow G P}=V_{\max , G Y \rightarrow G P} \times\left[\varepsilon\left(\frac{P S}{P S_{0}+P S}\right)+(1-\varepsilon)\left(\frac{C S}{C S_{0}+C S}\right)\right] ; \quad \varepsilon=0.75 \\
& V_{T G \rightarrow F A}=V_{\max , T G \rightarrow F A}\left(\frac{P S^{-1}}{P S_{0}^{-1}+P S^{-1}}\right) \\
& V_{P Y \rightarrow L A}=V_{\max , P Y \rightarrow L A} \times\left(\frac{R S}{R S_{0}+R S}\right) \\
& V_{P Y \rightarrow A C}=V_{\max , P Y \rightarrow A C} \times\left[\varepsilon\left(\frac{R S^{-1}}{R S_{0}^{-1}+R S^{-1}}\right)+(1-\varepsilon)\left(\frac{A F^{-1}}{A F_{0}^{-1}+A F^{-1}}\right)\right] ; \quad \varepsilon=0.75 \\
& V_{L A \rightarrow P Y}=V_{\max , L A \rightarrow P Y} \times\left(\frac{R S^{-1}}{R S_{0}^{-1}+R S^{-1}}\right) \\
& V_{A C \rightarrow C O 2}=V_{\max , A C \rightarrow C O 2} \times\left[\varepsilon\left(\frac{R S^{-1}}{R S_{0}^{-1}+R S^{-1}}\right)+(1-\varepsilon)\left(\frac{P S}{P S_{0}+P S}\right)\right] ; \quad \varepsilon=0.7 \\
& V_{02 \rightarrow H 2 O}=V_{\max , O} \rightarrow H 2 O \\
& V_{C R \rightarrow P C}=V_{\max , C R \rightarrow P C} \times\left(\frac{P S^{-1}}{P S_{0}^{-1}+P S^{-1}}\right) \\
& V_{P C \rightarrow C R}=V_{\text {max }, P C \rightarrow C R} \times\left(\frac{P S}{P S_{0}+P S}\right) \\
& V_{\text {CoAPool } \rightarrow F C}=V_{\max , \text { CoAPool } \rightarrow F C} \times\left(\frac{R S^{-1}}{R S_{0}^{-1}+R S^{-1}}\right) \times\left(\frac{1}{F C}\right) \\
& V_{A C \rightarrow \text { CoAPool }}=V_{\text {max }, A C \rightarrow \text { CoAPool }}\left(\frac{R S}{R S_{0}+R S}\right) \times\left(\frac{1}{\text { CoAPool }}\right) \\
& V_{\text {ATPhydolysis }}=V_{\max , \text { ATPhydolysis }}
\end{aligned}
$$


Table 2: Changes in Parameter Values

\begin{tabular}{|c|c|}
\hline Flux & $\left(\mu \mathrm{mol} \mathrm{g} \mathrm{g}^{-1} \mathrm{~min}^{-1}\right)$ \\
\hline$\phi_{\mathrm{CR} \rightarrow \mathrm{PC}}$ & 4.55 \\
\hline$\phi_{\mathrm{PC} \rightarrow \mathrm{CR}}$ & 4.55 \\
\hline Rate Coefficients & $\left(\mathrm{min}^{-1}\right)$ \\
\hline$V_{\max , G L \rightarrow G P}$ & 0.492 \\
\hline$V_{\max , F A \rightarrow T G}$ & 0.24 \\
\hline$V_{\text {max, } F A \rightarrow A C}$ & 0.944 \\
\hline $\mathrm{V}_{\max , \mathrm{GP} \rightarrow \mathrm{PY}}$ & 5.28 \\
\hline$V_{\max , G P \rightarrow G Y}$ & 1.6 \\
\hline$V_{\max , G Y \rightarrow G P}$ & 1.6 \\
\hline$V_{\max , T G \rightarrow F A}$ & 0.08 \\
\hline$V_{\text {max, } P Y \rightarrow L A}$ & 3.96 \\
\hline $\mathrm{V}_{\max , P Y \rightarrow A C}$ & 5.04 \\
\hline $\mathrm{V}_{\max , \mathrm{LA} \rightarrow P Y}$ & 3.84 \\
\hline $\mathrm{V}_{\max , \mathrm{AC} \rightarrow \mathrm{CO} 2}$ & 6.4 \\
\hline $\mathrm{V}_{\max , 02 \rightarrow \mathrm{H} 2 \mathrm{O}}$ & 5.63 \\
\hline$V_{\max , C R \rightarrow P C}$ & 100 \\
\hline $\mathrm{V}_{\max , \mathrm{PC} \rightarrow \mathrm{CR}}$ & 100 \\
\hline$V_{\max , A C \rightarrow C o A P o o l}$ & 0.247 \\
\hline$V_{\text {max }}$ CoAPool $\rightarrow F C$ & 0.051 \\
\hline $\mathrm{V}_{\max , \text { ATPhydrolysis }}$ & 34.9 \\
\hline $\mathrm{K}_{\mathrm{m}}$ Values & $\left(\mu \mathrm{mol} \mathrm{g} \mathrm{g}^{-1}\right)$ \\
\hline $\mathrm{K}_{\mathrm{m}, \mathrm{GL} \rightarrow \mathrm{GP}}$ & 0.025 \\
\hline $\mathrm{K}_{\mathrm{m}, \mathrm{FA} \rightarrow \mathrm{TG}}$ & 0.0245 \\
\hline $\mathrm{K}_{\mathrm{m}, \mathrm{FA} \rightarrow \mathrm{AC}}$ & 0.0245 \\
\hline $\mathrm{K}_{\mathrm{m}, \mathrm{GP} \rightarrow \mathrm{PY}}$ & 1.7 \\
\hline $\mathrm{K}_{\mathrm{m}, \mathrm{GP} \rightarrow \mathrm{GY}}$ & 0.17 \\
\hline $\mathrm{K}_{\mathrm{m}, \mathrm{GY} \rightarrow \mathrm{GP}}$ & 33 \\
\hline $\mathrm{K}_{\mathrm{m}, \mathrm{TG} \rightarrow \mathrm{FA}}$ & 4 \\
\hline $\mathrm{K}_{\mathrm{m}, \mathrm{PY} \rightarrow \mathrm{LA}}$ & 2 \\
\hline $\mathrm{K}_{\mathrm{m}, \mathrm{PY} \rightarrow \mathrm{AC}}$ & 0.2 \\
\hline $\mathrm{K}_{\mathrm{m}, \mathrm{LA} \rightarrow \mathrm{PY}}$ & 2 \\
\hline $\mathrm{K}_{\mathrm{m}, \mathrm{AC} \rightarrow \mathrm{CO} 2}$ & 0.0046 \\
\hline $\mathrm{K}_{\mathrm{m}, 02 \rightarrow \mathrm{H} 2 \mathrm{O}}$ & 0.01 \\
\hline $\mathrm{K}_{\mathrm{m}, \mathrm{CR} \rightarrow \mathrm{PC}}$ & 88 \\
\hline $\mathrm{K}_{\mathrm{m}, \mathrm{PC} \rightarrow \mathrm{CR}}$ & 88 \\
\hline $\mathrm{K}_{\mathrm{m}, \mathrm{AC} \rightarrow \text { CoAPool }}$ & 0.0046 \\
\hline $\mathrm{K}_{\mathrm{m}, \text { CoAPool } \rightarrow \mathrm{FC}}$ & 0.043 \\
\hline \multicolumn{2}{|c|}{ Weighting Coefficients } \\
\hline$\varepsilon_{\mathrm{GP} \rightarrow \mathrm{PY}}$ & eliminated \\
\hline$\varepsilon_{\mathrm{GP} \rightarrow \mathrm{GY}}$ & 0.75 \\
\hline$\varepsilon_{\mathrm{GY} \rightarrow \mathrm{GP}}$ & 0.75 \\
\hline$\varepsilon_{\mathrm{PY} \rightarrow \mathrm{AC}}$ & 0.75 \\
\hline$\varepsilon_{\mathrm{AC} \rightarrow \mathrm{CO} 2}$ & 0.7 \\
\hline
\end{tabular}

under normal conditions it is close to zero [19]. Incorporation of new elements in the previous model resulted in minor changes in a few "ping-pong" weighting coefficients, $\mathrm{K}_{\mathrm{m}}$ values, and rate coefficients (see Table 2). More- over, the results obtained with the improved model agreed well with those from the original model when simulating the metabolic response to a $60 \%$ reduction in myocardial blood flow [17].

Computer simulations (in silico experiments) of the metabolic responses to reduced myocardial blood flow (parameter MBF) were performed by solving the model equations using an implicit integrator, LSODES [21]. Simulation results from these parametric studies enable us to analyze the effects of myocardial ischemia of various degrees and durations. Experimentally, the extent of ischemia can be controlled by using an external circuit with a pump or an occlusion device on the left anterior descending coronary artery. In both cases, oxygen delivery to the myocardium is proportionate to the reduction in blood flow. In the present investigation, we used our computational model to simulate the metabolic responses to myocardial ischemia of various degrees. The severity of ischemia was set by reducing myocardial blood flow $\left(\mathrm{MBF}^{*}\right)$ from its normal value $1.0 \mathrm{ml} \mathrm{g}^{-1} \mathrm{~min}^{-1}$ to $0.1 \mathrm{ml} \mathrm{g}$ ${ }^{1} \mathrm{~min}^{-1}$ in $0.1 \mathrm{ml} \mathrm{g}^{-1} \mathrm{~min}^{-1}$ decrements. The reduction from normal to reduced blood flow was implemented exponentially over $1 \mathrm{~min}$. Due to methodological limitations, scarce data have been collected during the first few minutes and after 90 minutes of myocardial ischemia in in vivo animal models, therefore we ran the simulations out to 6 hours.

Since our overall goal was to investigate the regulation of glycolysis and lactate formation at the onset of ischemia and during the transition to a new metabolic steady state, we simulated the time profiles of (a) myocardial ATP, PCr, $\mathrm{NADH}, \mathrm{NAD}^{+}$, glycogen, pyruvate and lactate concentrations, (b) the rates of glucose, lactate, and oxygen uptake, and (c) the rates of glycolysis, lactate production, and pyruvate oxidation and focused our analysis on the early and late phases of the acute response to reduced blood flow. The transient responses were analyzed using nonlinear regression analysis to characterize the time profiles of the concentrations and flux rates changes due to ischemia. Response times $(\tau)$ were estimated from this analysis as the time it took to reach $\sim 63 \%$ of the steady state response change.

\section{Results}

Transient responses to myocardial blood flow reductions

The metabolic responses to reduced myocardial blood flow were evaluated by performing a series of computer simulations, each corresponding to a specific reduction in myocardial blood flow. Accordingly, myocardial blood flow was reduced from its normal value of 1.0 to $0.1 \mathrm{ml} \mathrm{g}$ ${ }^{1} \mathrm{~min}^{-1}$ in $10 \%$ decrements. For clarity, we only show the responses for myocardial blood flows of $0.8,0.6,0.4,0.2$ and $0.1 \mathrm{ml} \mathrm{g}^{-1} \mathrm{~min}^{-1}$. 
Flow reductions smaller than $0.2 \mathrm{ml} \mathrm{g}^{-1} \mathrm{~min}^{-1}$ from normal myocardial blood flow did not alter myocardial oxygen consumption $\left(\mathrm{MVO}_{2}\right)$ but resulted in small decreases $(<4 \%)$ in ATP and PCr concentrations, and moderate increases $(<10 \%)$ in $\mathrm{NADH} / \mathrm{NAD}^{+}$. Larger reductions in myocardial blood flow resulted in a rapid exponential decrease in $\mathrm{MVO}_{2}$ with $\tau$ ranging from $0.4 \mathrm{~min}$ at a flow of $0.1 \mathrm{ml} \mathrm{g}^{-1} \mathrm{~min}^{-1}$ to $0.9 \mathrm{~min}$ at a flow of $0.7 \mathrm{ml} \mathrm{g}^{-1} \mathrm{~min}^{-1}$ (Fig. 2A). The drop in $\mathrm{MVO}_{2}$ to new steady-state values was proportional to the degree of flow reduction, which has also been shown experimentally [22].

The redox state increased almost as rapidly as $\mathrm{MVO}_{2}$ decreased with $\tau$ ranging from $0.6 \mathrm{~min}$ at a flow of $0.1 \mathrm{ml}$ $\mathrm{g}^{-1} \mathrm{~min}^{-1}$ to $2.4 \mathrm{~min}$ at a flow of $0.7 \mathrm{ml} \mathrm{g}^{-1} \mathrm{~min}^{-1}$. Flow reductions beyond $0.8 \mathrm{ml} \mathrm{g}^{-1} \mathrm{~min}^{-1}$ also caused rapid increases in NADH content (concentration ratio of $\left.\mathrm{NADH} /\left(\mathrm{NADH}+\mathrm{NAD}^{+}\right)\right)$and a rapid fall in ATP $(\tau$ ranging from $0.7 \mathrm{~min}$ at a flow of $0.1 \mathrm{ml} \mathrm{g}^{-1} \mathrm{~min}^{-1}$ to $1.9 \mathrm{~min}$ at a flow of $0.8 \mathrm{ml} \mathrm{g}^{-1} \mathrm{~min}^{-1}$ ) to stable levels that were proportional to the fall in $\mathrm{MVO}_{2}$ (Figs. 2B and 2D). Phosphocreatine concentration fell less rapidly than ATP concentration ( $\tau$ ranging from $1.5 \mathrm{~min}$ at a flow of $0.1 \mathrm{ml}$ $\mathrm{g}^{-1} \mathrm{~min}^{-1}$ to $3.0 \mathrm{~min}$ at a flow of $0.8 \mathrm{ml} \mathrm{g}^{-1} \mathrm{~min}^{-1}$ ) and reached steady-state values nonlinearly related to the fall in $\mathrm{MVO}_{2}$ (Fig. 2C).

Lactate concentration and the lactate to pyruvate ratio (LA/PY) displayed a biphasic behavior in response to flow reductions beyond $0.8 \mathrm{ml} \mathrm{g}^{-1} \mathrm{~min}^{-1}$. Flow reductions caused a large (up to $26 \mu \mathrm{mol} \mathrm{g}^{-1}$ ) but slow ( $\tau$ ranging from $15 \mathrm{~min}$ at a flow of $0.8 \mathrm{ml} \mathrm{g}^{-1} \mathrm{~min}^{-1}$ to $38 \mathrm{~min}$ at a flow of $0.1 \mathrm{ml} \mathrm{g}^{-1} \mathrm{~min}^{-1}$ ) transient increase in lactate concentration followed by a slow exponential decrease toward steady-state levels (Fig. 2E) and a rapid decrease in LA/PY followed by a nonlinear increase toward steadystate values proportional to the reduction in myocardial blood flow (Fig. 2F).

The rate of glucose uptake decreased by half immediately $(\sim 24 \mathrm{sec})$ after the onset of blood flow reduction and then increased rapidly $(1.3 \mathrm{~min}<\tau<1.9 \mathrm{~min})$ toward steady state, with a peak rate at a flow of $0.2 \mathrm{ml} \mathrm{g}^{-1} \mathrm{~min}^{-1}$, and lower steady state values at a flow of $0.1 \mathrm{ml} \mathrm{g}^{-1} \mathrm{~min}^{-1}$ (Fig. $3 \mathrm{~A})$. The net rate of glycogen breakdown reached a peak after 5 to 7 minutes of flow reduction, and gradually returned toward zero by 120 to 180 minutes, and did not change out to 6 hours (Fig. 3B). The rate of glycolysis increased rapidly ( $\tau$ ranging from $1.7 \mathrm{~min}$ at a flow of 0.1 $\mathrm{ml} \mathrm{g}^{-1} \mathrm{~min}^{-1}$ to $5.8 \mathrm{~min}$ at a flow of $0.8 \mathrm{ml} \mathrm{g}^{-1} \mathrm{~min}^{-1}$ ) with the onset of flow reduction, peaking between 5 and 10 minutes after the onset, and then declined gradually up to $\sim 180$ minutes (Fig. 3C) as glycogen stores were depleted. The contribution of glycogen breakdown to the total rate of glycolysis over the initial 90 minutes was greater with more severe flow reduction. After approximately $120 \mathrm{~min}$ utes of ischemia net glycogen breakdown was minimal and glucose uptake was the primary provider of glycolytic substrate (Figs. 3A and 3B).

Under normal flow conditions there is net lactate uptake (i.e., a negative net lactate release) and a net rate of lactate oxidation by the heart. Simulation results indicated that in the early transient phase, net lactate release increased in proportion to the severity of the reduction in myocardial blood flow, with a peak effect $~ 13-28$ minutes after the onset of flow reduction. Then, net lactate release decreased exponentially toward steady levels, which were reached after approximately 150 minutes of flow reduction (Fig. 3D). The rate of lactate production increased at a faster rate (peak at $\sim 7.5 \mathrm{~min}$ after the onset of flow reduction) than the rate of lactate release, and was larger than the rate of lactate utilization at myocardial blood flows lower than $0.7 \mathrm{ml} \mathrm{g}^{-1} \mathrm{~min}^{-1}$ resulting in net lactate production and lactate release.

\section{Metabolic changes as a function of myocardial blood flow}

To determine to what extent the rate of glycolysis and lactate formation in the transition from normal to ischemic conditions are controlled by the supply of glycolytic substrate or the cellular redox and phosphorylation states, we examined the ischemia-induced changes in key concentrations and fluxes as a function of myocardial blood flow. Figures 4 and 5 are plots of the metabolic data as a function of myocardial blood flow during the early phase (at $7 \mathrm{~min}$ ) and after 30, 90 and 180 minutes of flow reduction.

Myocardial oxygen consumption remained constant when blood flow was reduced from 1.0 to $0.9 \mathrm{ml} \mathrm{g}^{-1} \mathrm{~min}^{-}$ ${ }^{1}$ but decreased linearly with blood flow reductions from 0.8 to $0.1 \mathrm{ml} \mathrm{g}^{-1} \mathrm{~min}^{-1}$ (Fig. $4 \mathrm{~A}$ ). The concentration ratio of $\mathrm{NADH} /\left(\mathrm{NADH}+\mathrm{NAD}^{+}\right)$increased exponentially as myocardial blood flow was reduced and oxygen consumption fell; NADH concentration values after 7 minutes of the onset of blood flow reduction were larger than those after the steady state was reached ( $180 \mathrm{~min}$ ) only when myocardial blood flow was between 0.2 and $0.6 \mathrm{ml} \mathrm{g}^{-1} \mathrm{~min}^{-1}$ (Fig. 4B). Myocardial ATP content did not change $(<1 \%)$ from its initial value when flow was decreased from 1 to $0.9 \mathrm{ml} \mathrm{g}^{-1} \mathrm{~min}^{-1}$, but it fell as a linear function of blood flow from 0.8 to $0.1 \mathrm{ml} \mathrm{g}^{-1} \mathrm{~min}^{-1}$ (Fig. 4C). On the other hand, phosphocreatine ( $\mathrm{PCr}$ ) content changed slightly $(<4 \%)$ when flow was reduced from 1.0 to $0.9 \mathrm{ml} \mathrm{g}^{-1} \mathrm{~min}^{-}$ 1 , but decreased exponentially to nearly zero when myocardial blood flow was reduced from 0.8 to $0.1 \mathrm{ml} \mathrm{g}^{-1} \mathrm{~min}^{-}$ 1 , reflecting its role as a "buffer" for ATP during the transition to reduced myocardial blood flow (Fig. 4D). Lactate concentration in the tissue increased exponentially with myocardial blood flow reduction, with the largest increase 

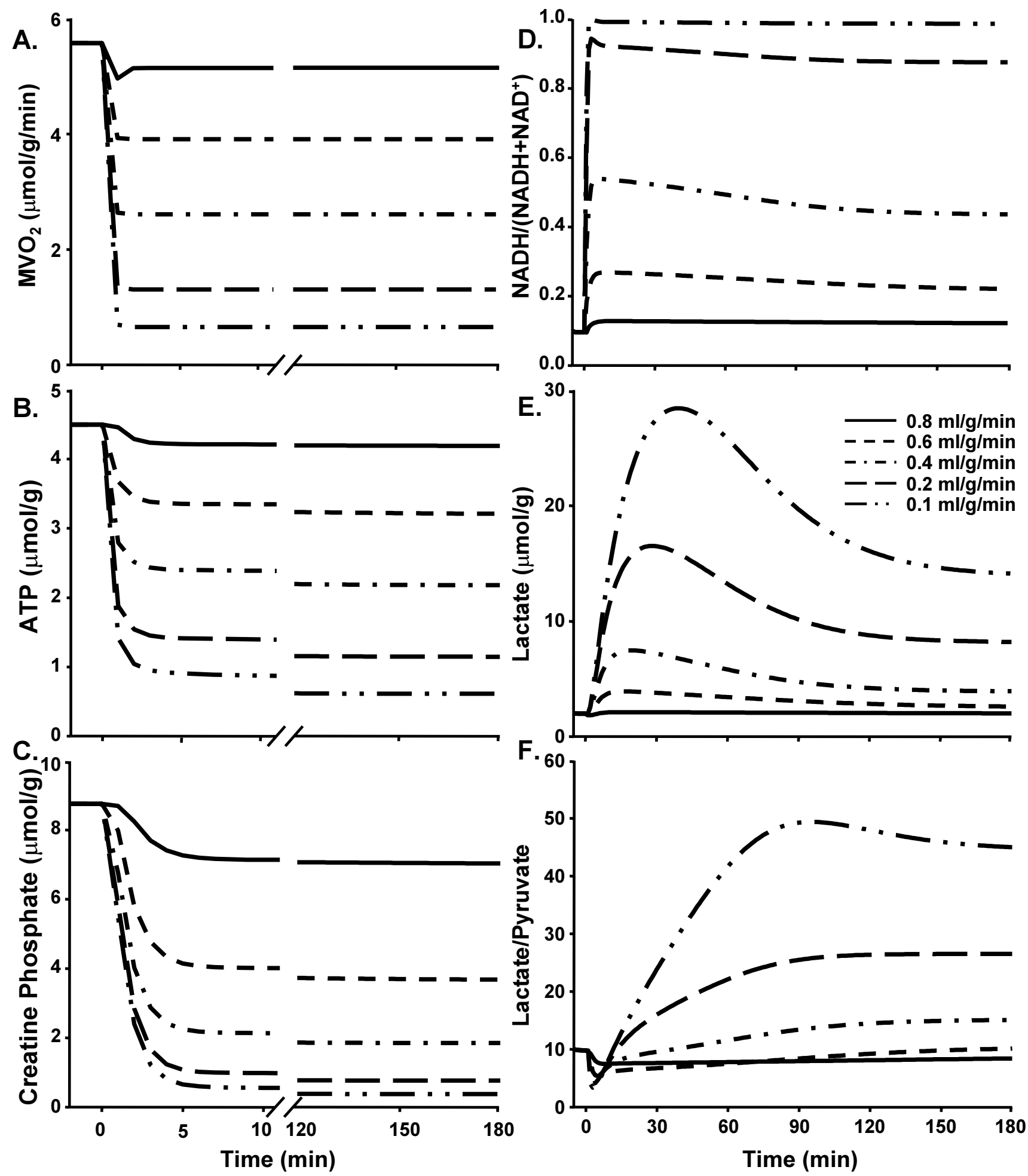

Figure 2

Time courses of myocardial (A) oxygen consumption $\left(\mathrm{MVO}_{2}\right),(\mathrm{B})$ ATP concentration, $(\mathrm{C})$ phosphocreatine concentration, (D) the concentration ratio of $\mathrm{NADH} /(\mathrm{NADH}+\mathrm{NAD})$, (E) lactate concentration, and (F) lactate to pyruvate ratio (LA/PY) from computer simulations of reductions $(0.8,0.6,0.4,0.2$, and $0.1 \mathrm{ml} / \mathrm{g} / \mathrm{min})$ in myocardial blood flow. 

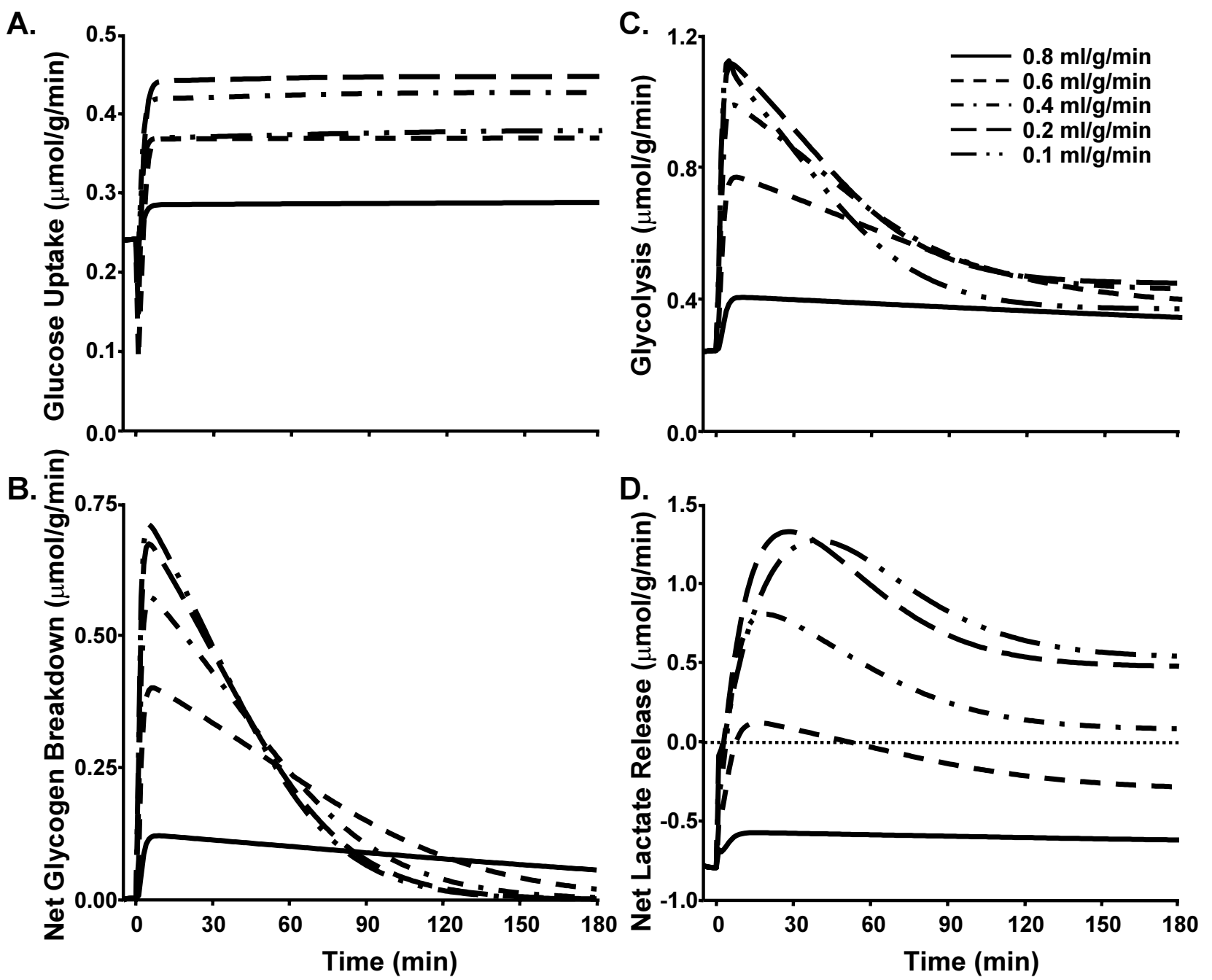

Figure 3

Dynamics of the myocardial rates of $(A)$ exogenous glucose uptake, (B) net glycogen breakdown, (C) glycolysis, and (D) net lactate release obtained from computer simulations of reductions $(0.8,0.6,0.4,0.2$, and $0.1 \mathrm{ml} / \mathrm{g} / \mathrm{min})$ in $\mathrm{myocardial}$ blood flow.

occurring after 30 min of the onset of flow reduction; lactate concentration decreased gradually thereafter, reaching a stable concentration by 180 minutes of flow reduction (Fig. 4E). The tissue lactate to pyruvate ratio did not change when blood flow was reduced from 1.0 to 0.8 $\mathrm{ml} \mathrm{g}^{-1} \mathrm{~min}^{-1}$, but increased nonlinearly with myocardial blood flow reductions from 0.7 to $0.1 \mathrm{ml} \mathrm{g}^{-1} \mathrm{~min}^{-1}$. This ratio kept increasing after 30 minutes of the onset of flow reduction, and reaching a steady value by 180 minutes (Fig. 4F).

The net rate of glycogen breakdown increased nonlinearly with myocardial blood flow reductions from 0.8 to $0.1 \mathrm{ml}$ $\mathrm{g}^{-1} \mathrm{~min}^{-1}$ after 7 and 30 minutes of the onset of flow reduction, while it only displayed a small increase followed by a gradual decrease after 90 minutes (Fig. 5A), reflecting the effects of glycogen depletion early in the ischemic period. The rate of glucose uptake increased nonlinearly with blood flow reduction, reaching a plateau 1.8 -fold above its normal value at $0.2 \mathrm{ml} \mathrm{g}^{-1} \mathrm{~min}^{-1}$. The rate of glucose uptake fell modestly when flow was further reduced to $0.1 \mathrm{ml} \mathrm{g}^{-1} \mathrm{~min}^{-1}$ (Fig. 5B).

Under normal aerobic conditions there was simultaneous conversion of lactate to pyruvate and pyruvate to lactate in the myocardium with net lactate uptake from the blood. 

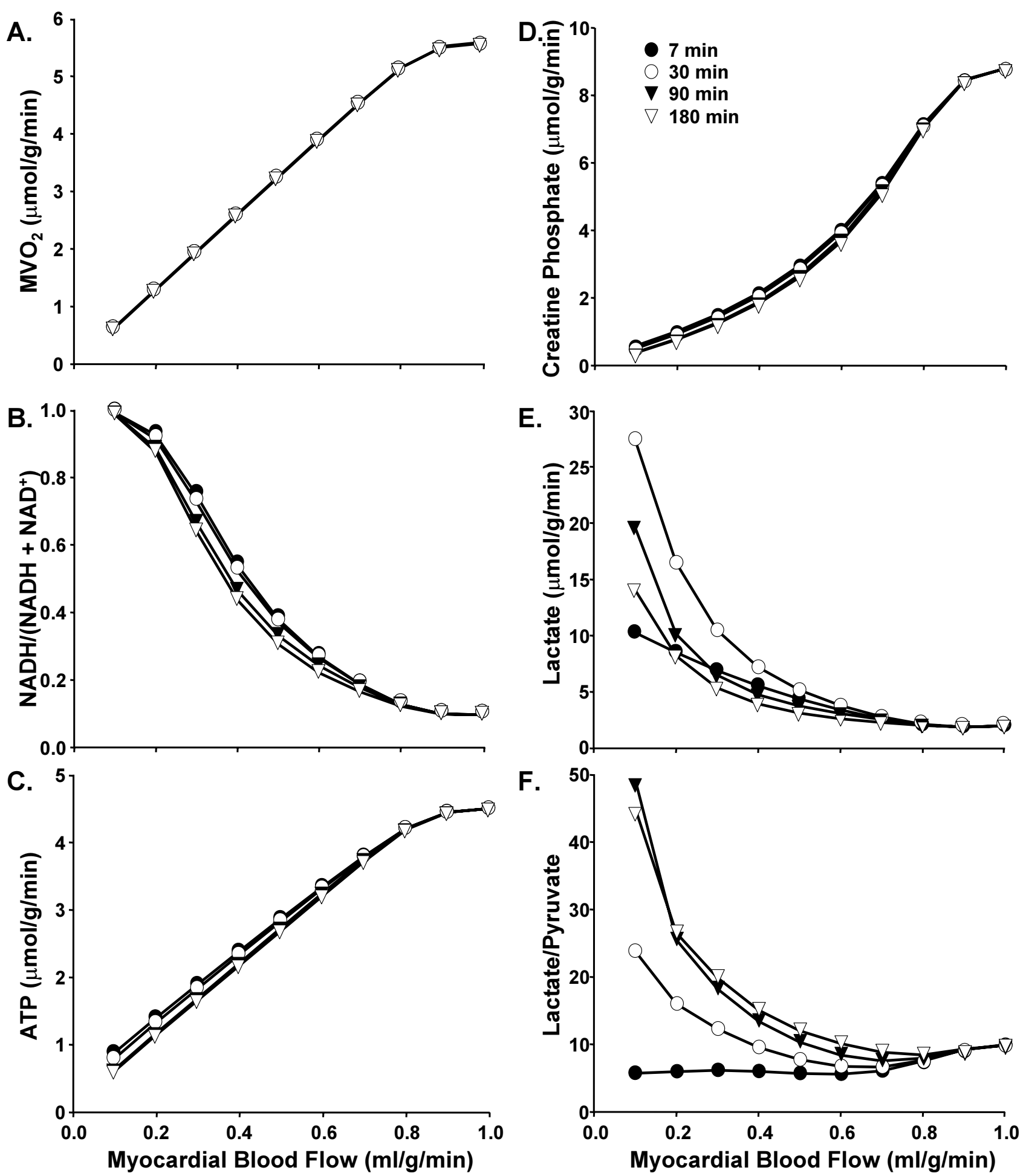

\section{Figure 4}

Computer-simulated values for myocardial (A) $\mathrm{MVO}_{2}$, (B) the concentration ratio of NADH/(NADH + NAD+), (C) ATP concentration, (D) phosphocreatine concentration, (E) lactate concentration, and (F) lactate to pyruvate ratio (LA/PY) after 7, 30, 90 , and 180 minutes of the onset of flow reduction plotted as a function of the myocardial blood flow. 

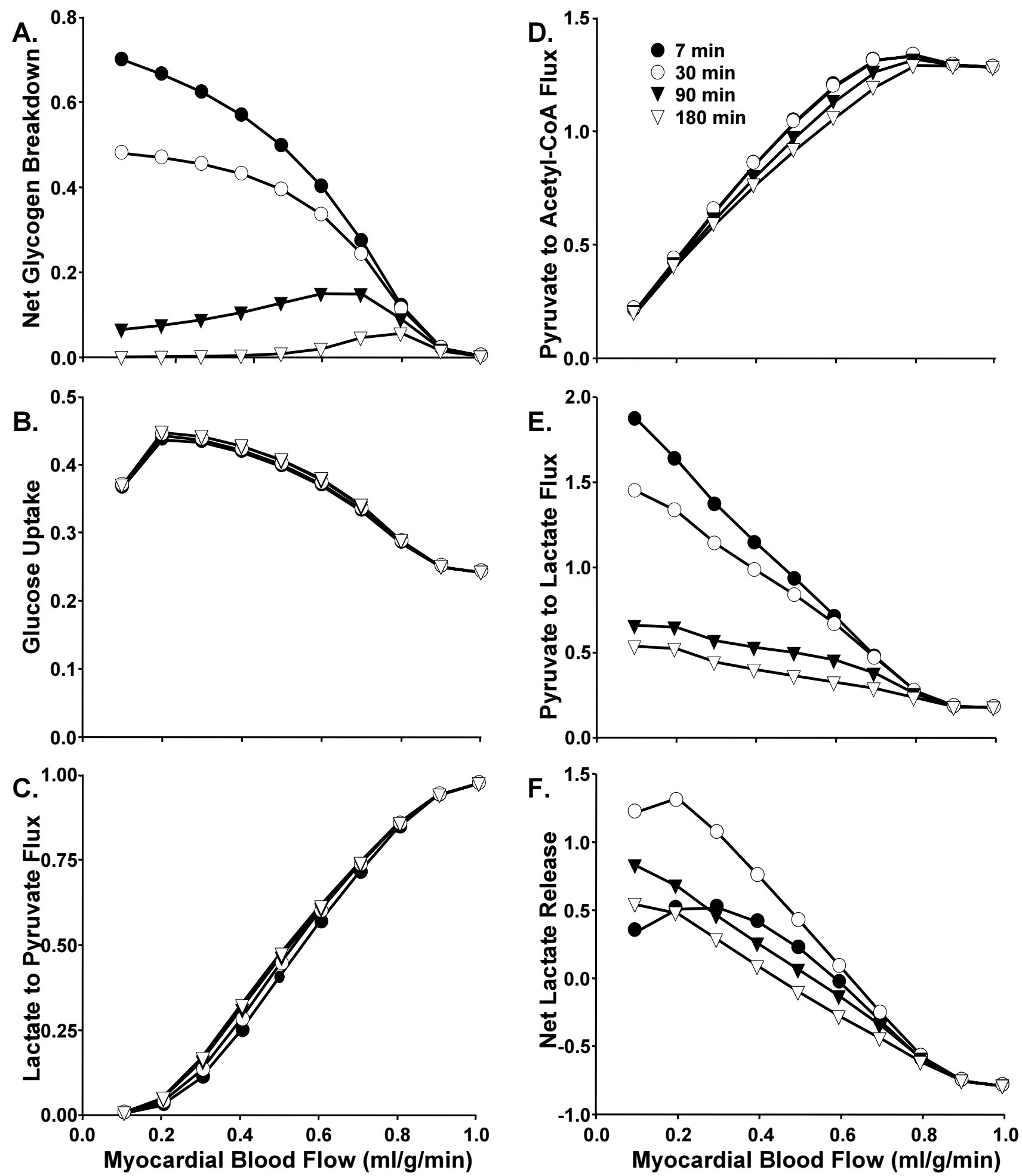

Figure 5

Computer-simulated values for myocardial rates of $(A)$ net glycogen breakdown, $(B)$ exogenous glucose uptake, (C) conversion of lactate to pyruvate, (D) pyruvate oxidation (conversion of pyruvate to acetyl-CoA), (E) conversion of pyruvate to lactate, and $(F)$ net lactate release after $7,30,90$, and 180 minutes of the onset of flow reduction plotted as a function of the myocardial blood flow. 
The rate of lactate conversion to pyruvate decreased linearly from 0.85 to $0.15 \mu \mathrm{mol} \mathrm{g}{ }^{-1} \mathrm{~min}^{-1}$ with blood flow reductions from 0.8 to $0.3 \mathrm{ml} \mathrm{g}^{-1} \mathrm{~min}^{-1}$, and was effectively zero with flows of $0.2 \mathrm{ml} \mathrm{g}^{-1} \mathrm{~min}^{-1}$ and less (Fig. 5C). On the other hand, the rate of pyruvate conversion to lactate increased linearly with myocardial blood flow reductions below $0.8 \mathrm{ml} \mathrm{g}^{-1} \mathrm{~min}^{-1}$, with a peak effect at $\sim 7.5$ minutes, and a progressive fall over 90 minutes, reaching stable values at 180 minutes (Fig. 5E). The net lactate release by the myocardium was negative under normal flow conditions, did not change much at flows of 0.9 and $0.8 \mathrm{ml} \mathrm{g}^{-1} \mathrm{~min}^{-1}$, and increased progressively with flows between 0.8 and $0.1 \mathrm{ml} \mathrm{g}^{-1} \mathrm{~min}^{-1}$ (Fig. 5F). The slope in the relationship between net lactate production and myocardial blood flow fell progressively from 7.5 minutes to 180 minutes and then remained constant out to 6 hours. The rate of pyruvate oxidation to acetyl-CoA decreased linearly with blood flow reductions between 0.7 and $0.1 \mathrm{ml} \mathrm{g}^{-1} \mathrm{~min}^{-1}$ (Fig. 5D).

The rate of glycolysis increased nonlinearly with myocardial blood flow reduction, and reached a plateau with flows between 0.3 and $0.2 \mathrm{ml} \mathrm{g}^{-1} \mathrm{~min}^{-1}$ after 7 and 30 minutes of the onset of flow reduction and between 0.6 and $0.2 \mathrm{ml} \mathrm{g}^{-1} \mathrm{~min}^{-1}$ after 90 minutes (Fig. 4C).

\section{Discussion}

Computer simulations of the transition from normal to reduced myocardial blood flow were used to study covariations between the rates of glycolysis and lactate formation and (1) the concentrations of NADH, NAD, ADP, and ATP and (2) the rates of glycogen breakdown and glucose uptake in order to elucidate mechanisms regulating anaerobic glycolysis in ischemic myocardium. Consequently, the time courses of the various processes affecting glycolysis and lactate dynamics from the onset of flow reduction and up to six hours later were computed and characterized for decreasing values of myocardial blood flow. Our results show that the glycolytic rate during the transition to reduced myocardial flow is regulated by glycogen breakdown, glucose uptake, ATP/ADP, and $\mathrm{PCr} / \mathrm{Cr}$. Two distinct mechanisms regulating myocardial lactate dynamics during flow reduction became apparent: (1) control by the redox state immediately after the onset of flow reduction and (2) control of glycolysis by substrate availability (i.e., glycogen) through the transition to a new steady state.

\section{Control of glycolysis during reduced myocardial blood flow} Examination of simulations show the crucial role of (1) $\mathrm{ATP} / \mathrm{ADP}$ and $\mathrm{PCr} / \mathrm{Cr}$ in the burst of glycolytic rate with the initiation of ischemia, and (2) glycolytic substrate supply in the later phase of the ischemic response. The time courses of ATP and PCr indicate that these metabolites contribute to the initial increase in the rates of glyco- lysis and glycogenolysis, respectively. Our modelsimulated results predicted PCr concentration falling less rapidly than ATP concentration, showing a sluggish response of PCr in buffering ATP. It is difficult to validate this prediction with experimental data from human-like animals, as limited data exists on the dynamic changes of ATP and PCr. Most data collected from these animal experiments have been collected after 5 minutes of the introduction of ischemia. The only studies that measured PCr and ATP using an in vivo preparation, showed decreases in both after 5 min of ischemia and while there were then no significant changes in ATP concentration after $5 \mathrm{~min}$ the results showed a regeneration of $\mathrm{PCr}$ after $5 \mathrm{~min}[1,3]$. It is clear from these results that more information on the dynamic changes of PCr and ATP concentrations is necessary in order to get a clear picture of their dynamic changes. However, given the known characteristics of the creatine kinase reaction [23] and the observed PCr and ATP dynamic responses from NMR studies in cardiac and skeletal muscle, it is apparent that the response time of $\mathrm{PCr}$ breakdown should have been smaller than that of ATP. Experimental studies by Gustafson and Kroll, using an isolated perfused rabbit heart subjected to a $95 \%$ reduction in flow $[23,24]$, showed that PCr decreased much quicker than ATP while their decreases $(75-78 \%$ and $46 \%$ for $\mathrm{PCr}$ and ATP, respectively) were much lower than our model predicts $(96 \%$ and $86 \%$ ). Therefore, our model, in its current form, does not appropriately represent the creatine kinase reaction and is unable to accurately predict PCr and ATP time courses at the onset of ischemia $(0-5 \mathrm{~min})$.

A plausible reason for the sluggishness in the $\mathrm{PCr}$ response to ischemia could be related to the ADP source in the model. Myocardial tissue biopsies taken from in vivo whole animal preparations are analyzed for total ADP concentration, which includes bound and free ADP. Since free ADP, instead of total ADP, participates in the control of myocardial respiration and glycogenolysis, future versions of this model should incorporate free ADP. As of today, we can only derive free ADP concentration from magnetic resonance spectroscopy data which are difficult to obtain in large animal in vivo preparations.

In the model, changes in ATP and PCr, mainly determine the initial direction of the change in fluxes via feedback, and to a lesser extent the magnitude of the changes in the rates of glycolysis and glycogenolysis. Lower ATP/ADP and $\mathrm{PCr} / \mathrm{Cr}$ values than those found under normal flow conditions result in higher glycolytic and glycogenolytic flux rates, while higher ATP/ADP and $\mathrm{PCr} / \mathrm{Cr}$ values have the opposite effect. The magnitude of the increase in the glycolytic rate though, depends primarily on the net rate of glycogen breakdown and to a lesser extent on glucose uptake and reduced ATP/ADP and $\mathrm{PCr} / \mathrm{Cr}$ values. The rate 
of glycogen breakdown increases several fold immediately after the onset of flow reduction increases up to $\sim 2$-fold. The ATP/ADP and PCr/Cr ratios decreased almost immediately after the onset of flow reduction and remained constant thereafter. Analysis of the time profiles of the covariations of the glycolytic rate and the above mentioned factors suggests that all contribute to the increase in glycolysis during the early transient phase following flow reduction; however, only glycogen breakdown seems to control the rate of glycolysis beyond 10 minutes.

\section{Control of lactate production and release during reduced myocardial blood flow}

Simulated metabolic responses showed a rapid increase in the rate of lactate production, at the onset of flow reduction, which declined to a new steady state value after $\sim 90$ to $180 \mathrm{~min}$, as previously demonstrated in swine myocardium $[9,11]$. Our results also indicated that the rate of glycolysis was the only flux rate that increased faster than the rate of lactate production. The redox state $\left(\mathrm{NADH} / \mathrm{NAD}^{+}\right)$ also changed fast enough to contribute to the large increase in lactate production. However, after the initial phase following the onset of flow reduction, both the rates of lactate production and glycolysis decreased gradually as linear functions of time while the redox state remained almost constant or decreased very slowly. The rates of glycolysis and net lactate release increased sharply in a flow dependent manner with the onset of flow reduction, and gradually returned to new steady-state values after 90 minutes. In contrast, the concentration ratio of $\mathrm{NADH} /\left(\mathrm{NADH}+\mathrm{NAD}^{+}\right)$increased sharply when flow was decreased, and remained elevated thereafter (Fig. 2D). Thus, the transient burst of lactate production at the onset of flow reduction was primarily the result of a rapid acceleration of glycolysis and, an increase in the cellular redox state which was mainly determined by the drop in $\mathrm{MVO}_{2}$. The fall in the rates of glycolysis and lactate production from 15-120 minutes was not due to changes in redox or phosphorylation state, but rather to glycogen depletion and a switch to dependence on exogenous glucose for glycolytic substrate.

In our model, the fall in myocardial blood flow and oxygen consumption result in a decrease in ATP and PCr concentrations, which activate glycogenolysis, glycolysis, and glycolytic pyruvate formation [17]. Although the intracellular regulation of glycogenolysis occurs by way of $P_{i}$ in our model the ratio of $\mathrm{PCr} / \mathrm{Cr}$ is used as a regulator, instead of $P_{\mathrm{i}}$. Because $\mathrm{P}_{\mathrm{i}}$ is not explicitly considered in this version of the model, the $\mathrm{PCr} / \mathrm{Cr}$ ratio is the best current approximation of $P_{i}$. Future models should incorporate $P_{i}$ to describe more accurately this regulation process.

The decrease in $\mathrm{MVO}_{2}$ with ischemia results in a parallel decrease in redox state which remains relatively constant throughout the remainder ischemia (Fig. 5D). The decrease in pyruvate oxidation, on the other hand, is not in parallel with the decrease in $\mathrm{MVO}_{2}$ due to the sudden increase in glycolytically-derived pyruvate formation during the first few minutes of ischemia (Figs. 4A and 5D). This suggests that once $\mathrm{MVO}_{2}$ is reduced due to underperfusion, the redox state increases rapidly to control the relative proportion of NADH that is oxidized via lactate formation or oxidative phosphorylation (Figs. 2A,2B). A redox shift towards reduction, in the presence of insufficient blood flow and increased glycolysis, would either decrease the rate of pyruvate conversion to acetyl-CoA or keep it constant (Fig. 5D), thus increasing the rate of lactate production (Fig. 5E). Therefore, while the extent of lactate or water formation coupled to NADH oxidation is mainly determined by the redox state right after the onset of flow reduction, the magnitude of the increase in lactate production with ischemia is mainly determined by the rate of glycolysis throughout the entire period of flow reduction.

In this model we used a top-down approach which focuses on integration and incorporation of those elements which are essential in predicting the metabolic responses to ischemia. Accordingly, at the initial stages of modeling cardiac metabolism, only key physiological aspects were included to reduce complexity and allow comparison with available in vivo data. As appropriate experimental data on dynamic responses become available, future models will incorporate additional elements as needed for testing hypotheses on the regulation of myocardial metabolism during stresses.

\section{Conclusion}

In summary, in silico studies were performed using a previously validated computational model of cardiac metabolism to examine the extent that glycolysis and lactate formation are controlled by the supply of glycolytic substrate and/or the cellular redox and phosphorylation states during the transition from normal to reduced blood flow conditions. Our results show that multiple timedependent mechanisms regulate the rates of glycolysis and lactate formation. Changes in phosphorylation state and glucose uptake affect primarily the initial phase of the glycolytic adaptation to ischemia, while glycogen breakdown exerts the main control over glycolysis during both the early transient phase and the late adaptation to ischemia. Similarly, changes in the redox state affect the rates of lactate formation and release primarily during the initial transient phase of the response to reductions in blood flow, while the rate of glycolysis controls the rate of lactate formation throughout the entire period of adaptation. 


\section{List of Abbreviations \\ GL Glucose}

FA Fatty Acid

GP Glucose-6-Phosphate

GY Glycogen

TG Triglyceride

PY Pyruvate

LA Lactate

AC Acetyl-CoA

FC Free-CoA

$\mathrm{O}_{2}$ Oxygen

$\mathrm{CO}_{2}$ Carbon Dioxide

$\mathrm{NAD}^{+}$Nicotinamide Adenine Dinucleotide (oxidized form)

NADH Nicotinamide Adenine Dinucleotide (reduced form)

AMP Adenosine Monophosphate

ADP Adenosine Diphosphate

ATP Adenosine Triphosphate

$\mathrm{P}_{\mathrm{i}}$ Inorganic Phosphate

Cr Creatine

PCr Phosphocreatine

$\mathrm{V}_{\mathrm{j}}$ Effective Volume

$\mathrm{C}_{\mathrm{aj}}$ Arterial Concentration of Species $\mathrm{j}$

$\sigma_{\mathrm{j}}$ Blood-Tissue Partition Coefficient for species j

$\mathrm{C}_{\mathrm{j}}$ Species $\mathrm{j}$ Concentration

LAD Left Anterior Descending Coronary Artery

MBF Myocardial Blood flow

$\mathrm{MVO}_{2}$ Myocardial Oxygen Consumption
$\mathrm{K}_{\mathrm{m}}$ Michaelis-Menten Constant

$\mathrm{V}_{\mathrm{kj}}$ Maximal Rate Coefficient

$\mathrm{V}_{\max }$ Michaelis-Menten Maximal Rate Coefficient

$\varepsilon$ Weighting Coefficient

AF AC/FC

CS Cr/PCr

RS NADH/NAD ${ }^{+}$

PS ADP/ATP

\section{Acknowledgements}

This work was supported in part by National Institute of Health Grant No. HL58653 and GM66309, American Heart Association Grant No.

$9660355 \mathrm{~V}$, and NASA-NSBRI Grant No. IHF00205. J.E.Salem was supported in part by a predoctoral training grant, NIH SIB Grant No. GM07535.

\section{References}

I. Arai AE, Pantely GA, Anselone CG, Bristow J, Bristow JD: Active downregulation of myocardial energy requirements during prolonged moderate ischemia in swine. Circ Res 1991, 69:|458-| 469.

2. McNulty PH, Sinusas AJ, Shi CQ, Dione D, Young LH, Cline GC, Shulman Gl: Glucose metabolism distal to a critical coronary stenosis in a canine model of low-flow myocardial ischemia. J Clin Invest 1996, 98:62-69.

3. Pantely GA, Malone SA, Rhen WS, Anselone CG, Arai A, Bristow J, Bristow JD: Regeneration of myocardial phosphocreatine in pigs despite continued moderate ischemia. Circ Res 1990, 67:|48|-|493.

4. Stanley WC, Hall JL, Smith KR, Cartee GD, Hacker TA, Wisneski JA: Myocardial glucose transporters and glycolytic metabolism during ischemia in hyperglycemic diabetic swine. Metabolism 1994, 43:61-69.

5. Stanley WC, Hernandez LA, Spires D, Bringas J, Wallace S, McCormack JG: Pyruvate dehydrogenase activity and malonyl CoA levels in normal and ischemic swine myocardium: effects of dichloroacetate. J Mol Cell Cardiol I996, 28:905-914.

6. Stanley WC, Hall JL, Stone CK, Hacker TA: Acute myocardial ischemia causes a transmural gradient in glucose extraction but not glucose uptake. Am J Physiol 1992, 262:H9l-H96.

7. Hall JL, Hernandez LA, Henderson J, Kellerman LA, Stanley WC: Decreased interstitial glucose and transmural gradient in lactate during ischemia. Basic Res Cardiol 1994, 89:468-486.

8. Neely JR, Whitmer JT, Rovetto MJ: Effect of coronary blood flow on glycolytic flux and intracellular $\mathrm{pH}$ in isolated rat hearts. Circ Res 1975, 37:733-74I.

9. Fedele FA, Gewirtz H, Capone RJ, Sharaf B, Most AS: Metabolic response to prolonged reduction of myocardial blood flow distal to a severe coronary artery stenosis. Circulation 1988, 78:729-735.

10. Liedtke AJ: Alterations of carbohydrate and lipid metabolism in the acutely ischemic heart. Prog Cardiovasc Dis 1981, 23:321-336.

II. Stanley WC, Lopaschuk GD, Hall JL, McCormack JG: Regulation of myocardial carbohydrate metabolism under normal and ischaemic conditions. Potential for pharmacological interventions. Cardiovasc Res 1997, 33:243-257.

12. Achs MJ, Garfinkel D: Computer simulation of energy metabolism in anoxic perfused rat heart. Am J Physiol 1977, 232:RI64-RI74. 
13. Ch'en FF, Vaughan-Jones RD, Clarke K, Noble D: Modelling myocardial ischaemia and reperfusion. Prog Biophys Mol Biol 1998, 69:515-538.

14. Kashiwaya Y, Sato K, Tsuchiya N, Thomas S, Fell DA, Veech RL, Passonneau JV: Control of glucose utilization in working perfused rat heart. J Biol Chem 1994, 269:25502-255 I4.

15. Jafri MS, Dudycha SJ, O'Rourke B: Cardiac energy metabolism: models of cellular respiration. Annu Rev Biomed Eng 200I, 3:57-8I.

16. Kohn MC, Garfinkel D: Computer simulation of entry into glycolysis and lactate output in the ischemic rat heart. J Mol Cell Cardiol 1978, 10:779-796.

17. Salem JE, Saidel GM, Stanley WC, Cabrera ME: Mechanistic model of myocardial energy metabolism under normal and ischemic conditions. Ann Biomed Eng 2002, 30:202-2I6.

18. Hall JL, Lopaschuk GD, Barr A, Bringas J, Pizzurro RD, Stanley WC: Increased cardiac fatty acid uptake with dobutamine infusion in swine is accompanied by a decrease in malonyl CoA levels. Cardiovasc Res 1996, 32:879-885.

19. Panchal AR, Comte B, Huang H, Kerwin T, Darvish A, Des Rosiers C Brunengraber $\mathrm{H}$, Stanley WC: Partitioning of pyruvate between oxidation and anaplerosis in swine hearts. Am J Physiol Heart Circ Physiol 2000, 279: $\mathrm{H} 2390-\mathrm{H} 2398$.

20. Principles of Metabolic Control in Mammaliaa Systems Edited by: HermanRH, CohnRM and NamaraPD. New York, Plenum Press; 1980:I-669.

21. Subroutine LSODES. 2004.

22. Ito BR: Gradual onset of myocardial ischemia results in reduced myocardial infarction. Association with reduced contractile function and metabolic downregulation. Circulation 1995, 9 I:2058-2070.

23. Kroll K, Kinzie DJ, Gustafson LA: Open-system kinetics of myocardial phosphoenergetics during coronary underperfusion. Am J Physiol 1997, 272: $\mathrm{H} 2563-\mathrm{H} 2576$.

24. Gustafson LA, Kroll K: Downregulation of 5'-nucleotidase in rabbit heart during coronary underperfusion. Am J Physiol 1998 , 274: $\mathrm{H} 529-\mathrm{H} 538$.
Publish with Bio Med Central and every scientist can read your work free of charge

"BioMed Central will be the most significant development for disseminating the results of biomedical research in our lifetime. "

Sir Paul Nurse, Cancer Research UK

Your research papers will be:

- available free of charge to the entire biomedical community

- peer reviewed and published immediately upon acceptance

- cited in PubMed and archived on PubMed Central

- yours - you keep the copyright
BioMedcentral 